\title{
Spatial optimality in power distribution networks
}

\author{
Lingen Luo \\ Department of Electrical Engineering \\ Shanghai Jiao Tong University \\ Shangai (China) \\ 1lg523@gmail.com \\ Giuliano A. Pagani \\ Distributed Systems Group \\ Rijksuniversiteit Groningen \\ Groningen (The Netherlands) \\ g.a.pagani@rug.nl \\ Marti Rosas-Casals \\ Sustainability Measurement and Modelling Lab \\ Universitat Politècnica de Catalunya - Barcelona Tech \\ Barcelona (Spain) \\ rosas@mmt.upc.edu
}

\begin{abstract}
Critical infrastructures can be characterized as networks where nodes and edges are embedded in space. Transportation networks, the internet, and power grids, are examples of networks where spatial constrains are relevant. An important consequence of space is that there is a cost associated with the length of edges which in turn has important effects on the topological structure of and on the dynamical processes which take place on these spatial networks. In this paper we investigate the effect of the wiring cost in the spatial organization of a sample of power distribution networks by means of shuffling the networks in systematic ways. We show that although they share similar topologies, suboptimal networks (i.e., those with topologies not organized to reduce the wiring cost) seem to accumulate more failures. Consequences of these results and further work are finally discussed and outlined.
\end{abstract}

Keywords-power distribution networks; spatial networks; optimality; wiring cost.

\section{INTRODUCTION}

The need of modeling systems with many interacting components organized in non-trivial topologies has given birth to a new approach of analyzing interconnected systems known as complex networks [1]. An example of such a complex system is the power grid. Power grids, especially transmission networks, have been widely studied applying the complex network approach. Usually, basic topological characteristics, statistical global graph properties and vulnerability (or robustness) analysis have been performed on many power grids in different parts of the world [2]. Especially, the vulnerability characteristic of the power grid is the main motivation for the studies. In fact topology property plays an important role in shaping the performance (e.g., effects of natural disasters or malicious attacks) of power grids [3-6]. As a result, there is an increasing interest in analyzing structural vulnerability of power grids by means of complex networks methodology.

In current power systems, power plants exploit economies of scales and more efficient technologies and are usually located far away from the load center. Power is then transmitted from power plants to load centers by high voltage transmission networks, and finally distributed to different voltage levels to users, like homes, offices, schools, stores, etc. Therefore, the power grid is usually divided in two main segments: transmission (high voltage) and distribution (low voltage) networks. Most of the scientific literature using the complex networks approach applied to the power grid has focused so far on transmission networks, while little attention has been paid on the distribution grid. Until to now, to our best knowledge, only Ref. [7] took distribution network into consideration under emerging smart grid technology. As addressed by the authors, with the development of the smart grid, the main role of high voltage transmission network may change while the low voltage distribution network may gain more and more importance and require a major update. Most of the research that focuses on modeling the power grid uses simple graph models with sometimes the use of basic properties such as direction and weight. However, these studies [2] miss an important characteristic of the power grid: the spatial characteristic. Spatial properties are basically the coordinates of the generators, transformers and substations, and the length of power cables.

Another key aspect of many practical engineering problems concerns optimization. Optimization can be applied also in the network context and usually the objective is to identify optimal networks or optimal network models and the optimal flow or traffic on a network [8-10]. Optimization in power systems is also an important topic such as, the optimal dispatch of power 
generation [11], the optimal method for power distribution network reconfiguration [12], the optimal placement of phasor measurement units and optimal control strategy for power system facility and stability, which covers from the static to dynamic analysis of power systems. Two key issues should be taken into consideration in the optimization of asset utilization, maintenance and replacement: performance and cost. In order to assess the performance of a power system from an engineering point of view, direct measurements representing real malfunction data like total loss of power, energy not supplied or restoration time, can be used [13]. The question we are assessing in this paper is how the performance of the network competes with its wiring cost, defined as the sum of the Euclidean length of power cables [14]. As in Ref. [14], we use two systematic ways to modify the structure of the networks and minimize the wiring cost function: edge exchange (EE) and vertex swapping (VS) shuffling methods. In the EE method [16] vertices of randomly selected two edges exchange their partner vertices. The degree (i.e., the number of edges incident to the vertex) of each vertex is unchanged and the positions of all vertices remain the same. In the VS method [17], two randomly chosen vertices simply exchange their positions while preserving all the connections. In this case the connection structure of the network never changes; however, the distances are altered as we repeat the process. As in Ref. [14], we adopt a Monte-Carlo (MC) scheme with simulated annealing, using the wiring cost as our Hamiltonian.

\section{Characteristics OF THE TOPOlOGY OF Distribution POWER GRIDS}

\section{A. Power grid data sets}

We have analyzed two kinds of power grids as spatial networks: transmission and the distribution power network. The transmission networks is a large scale interconnected bulk power transport grid. As a sample of this grid we use the pan European network known as Union for the Coordination of Transmission Electricity (UCTE). We use the UCTE network in order to compare the results of the distribution and the transmission layers of the power grid. In order to analyze the distribution network we use samples of distribution networks from Spain (S1 and S2) and The Netherlands (N1 to N12).

Formally, a complex network can be presented as a graph. In our abstraction to represent the power grid as an undirected graph $G=(N, E)$, consisting of two sets $N$ and $E$, such that $\mathrm{N} \neq \varnothing$ and $E$ is a set of unordered pairs of elements of $N$. The elements of $N \equiv\left\{n_{1}, n_{2} \ldots n_{N}\right\}$ are the nodes (or vertices, or points) of the graph $G$, while the elements of $E \equiv\left\{e_{1}, e_{2}, \ldots\right.$, $\left.e_{\mathrm{K}}\right\}$ are its links (or edges, or lines). We consider all the substations and transformers equal and they are presented as nodes in a graph and cables are abstracted as edges [15]. The basic information about these networks is reported in Table 1, where we have the number of nodes, the number of lines and the acronym of each distribution network analyzed.

\section{B. Topological metrics}

In the complex networks approach several metrics are used to quantify properties both from a global and local point of view [1]. The metrics of the several power grids are reported in Table 2, where average degree $<k>$, average shortest path length $L$, average clustering coefficient $C$ and graph density $<\rho>$ (as percentage) are shown.

TABLE I. Basic power grid data.

\begin{tabular}{|c|c|c|l|}
\hline $\begin{array}{c}\text { Network } \\
\text { type }\end{array}$ & N & L & Name / Geography \\
\hline Transmission & 2777 & 3762 & UCTE / Europe \\
Distribution & 519 & 557 & S1 / Spain \\
Distribution & 240 & 263 & S2 / Spain \\
Distribution & 451 & 492 & N1/ The Netherlands \\
Distribution & 473 & 505 & N2 / The Netherlands \\
Distribution & 241 & 254 & N3 / The Netherlands \\
Distribution & 287 & 305 & N4 / The Netherlands \\
Distribution & 221 & 231 & N5 / The Netherlands \\
Distribution & 193 & 209 & N6/ The Netherlands \\
Distribution & 957 & 1095 & N7 / The Netherlands \\
Distribution & 371 & 391 & N8 / The Netherlands \\
Distribution & 223 & 237 & N9 / The Netherlands \\
Distribution & 204 & 207 & N10/ The Netherlands \\
Distribution & 271 & 279 & N11/ The Netherlands \\
Distribution & 480 & 509 & N12/ The Netherlands \\
\hline
\end{tabular}

The degree (or connectivity) $k_{i}$ of a node $i$ is the number of edges incident with the node, and is defined in terms of the adjacency matrix $\mathbf{A}$ as:

$$
k_{i}=\sum_{j \in N} a_{i j}
$$

A measure of the typical separation between two nodes in the graph is given by the average shortest path length, also known as characteristic path length, defined as the mean of geodesic lengths over all couples of nodes:

$$
L=\frac{1}{N(N-1)} \sum_{i, j \in N, i \neq j} d_{i j}
$$

In terms of a generic graph, clustering means the presence of a high number of triangles. This can be quantified as the fraction of connected triples of nodes (triads) which also form triangles. Finally, the fraction of possible edges that exist in a graph is known as graph density:

$$
\rho=\frac{2 E}{N(N-1)}
$$

Results in Table 2 show that both, transmission and distribution networks, are very sparse graphs with similar topological values. Only the slightly higher value of the average degree for the UCTE transmission network could give us a hint of the more radial-like structure of the distribution grids, while the transmission networks present a more meshed topology.

\section{SPATIAL OPTIMALITY AND RELIABILITY}

The trade-off between performance and cost of a distribution network is a key issue. If connectivity is not a constraint, redundancy can be increased and the performance of a network can be greatly enhanced using sufficiently many edges. However, more edges imply more resources in all 
practical situations, and thus we would expect a competition between performance (measured as major failures or similar malfunctions) and cost (simply measured, in this case, as the sum of the Euclidean lengths of edges).

TABLE II. Basic topological results.

\begin{tabular}{|c|c|c|c|c|}
\hline $\begin{array}{c}\text { Network } \\
\text { name }\end{array}$ & $<\boldsymbol{k}>$ & $\boldsymbol{L}$ & $\boldsymbol{C}$ & $<\boldsymbol{\rho}\rangle$ \\
\hline UCTE & 2.71 & 22.7 & 0.07 & 0.10 \\
\hline S1 & 2.14 & 24.6 & 0.01 & 0.41 \\
\hline S2 & 2.19 & 15.8 & 0.00 & 0.92 \\
\hline N1 & 2.21 & 11.0 & 0.00 & 0.48 \\
\hline N2 & 2.15 & 17.0 & 0.01 & 0.45 \\
\hline N3 & 2.11 & 11.6 & 0.00 & 0.88 \\
\hline N4 & 2.18 & 12.7 & 0.01 & 0.74 \\
\hline N5 & 2.12 & 10.2 & 0.00 & 0.95 \\
\hline N6 & 2.17 & 9.2 & 0.00 & 1.13 \\
\hline N7 & 2.34 & 9.8 & 0.00 & 0.24 \\
\hline N8 & 2.11 & 15.0 & 0.00 & 0.57 \\
\hline N9 & 2.16 & 10.8 & 0.00 & 0.96 \\
\hline N10 & 2.05 & 15.6 & 0.00 & 1.00 \\
\hline N11 & 2.08 & 14.7 & 0.00 & 0.76 \\
\hline N12 & 2.15 & 13.1 & 0.00 & 0.44 \\
\hline
\end{tabular}

Real networks that can be viewed in such a way include electric circuits, the internet, the power grid, and the neuronal network in biological organisms, to name just a few. In this sense, low clustering coefficients for example ( $C$ in Table II) is the expected outcome of a distribution of electric power which avoids triangles in order to reach as much population as possible with minimum cost. Following Ref. [14], here we use the same procedure to investigate the role, if any, of the wiring cost in the performance of a power distribution network. Two systematic methods to shuffle the fifteen power networks' structure are used: (1) edge exchange (EE) and (2) vertex swapping (VS). In the EE method [16] vertices of randomly selected two edges exchange their partner vertices. The degree (Eq. 1) of each vertex remains unchanged and the positions of all vertices remain the same. In the VS method [17], two randomly chosen vertices simply exchange their positions while preserving all the connections. In this case the connection structure of the network never changes; however, the distances are altered as we repeat the process. Inspired by the method used in [14], we apply a Monte-Carlo (MC) scheme using the Metropolis algorithm controlled by a given "temperature" T (introduced only as an updating control parameter for the algorithm, and thus not related with any actual meaning of real temperature). The fully random shuffling of the network using either EE or VS method corresponds to the $\mathrm{MC}$ simulation at $\mathrm{T}=\infty$, and it has been defined as EE(inf) and VS(inf) respectively. Simulated annealing technique, starting from $\mathrm{T}=\infty$ and slowly decreasing it until $\mathrm{T}=0$ is reached, has been used to get the optimal value denoted as $\operatorname{EE}(0)$. The simulation results for the fifteen networks are shown in Figure 1, where EE(inf), EE(0) and VS(inf) have been all normalized by means of the original wiring length cost. We observe that all networks can be spatially optimized by method $\mathrm{EE}(0)$, but that methods VS(inf) and EE(inf) are not able to optimize the current spatial topology of the distribution networks except for one case: network S2, the only one which can be optimized by both $\mathrm{EE}(\mathrm{inf})$ and $\mathrm{EE}(0)$ methods.

The consequences of these results on the performance of the distribution networks are not an easy task. A common approach has been the correlation of major events (i.e., equivalent time of interruption, energy not supplied, restoration time, power loss, etc.) with some topological characteristic of the network (e.g., average degree $\langle k>$ in Table II), which has been shown useful in order to segregate European power transmission networks into fragile and robust ones [5]. Although a similar procedure can be applied to distribution networks, access to data is highly restricted. Whereas transmission system operators (TSO's) are forced to inform UCTE/ENTSO about major events on their grids, distribution systems operators (DSO's) are not. This fact poses a difficult drawback on this kind of research.

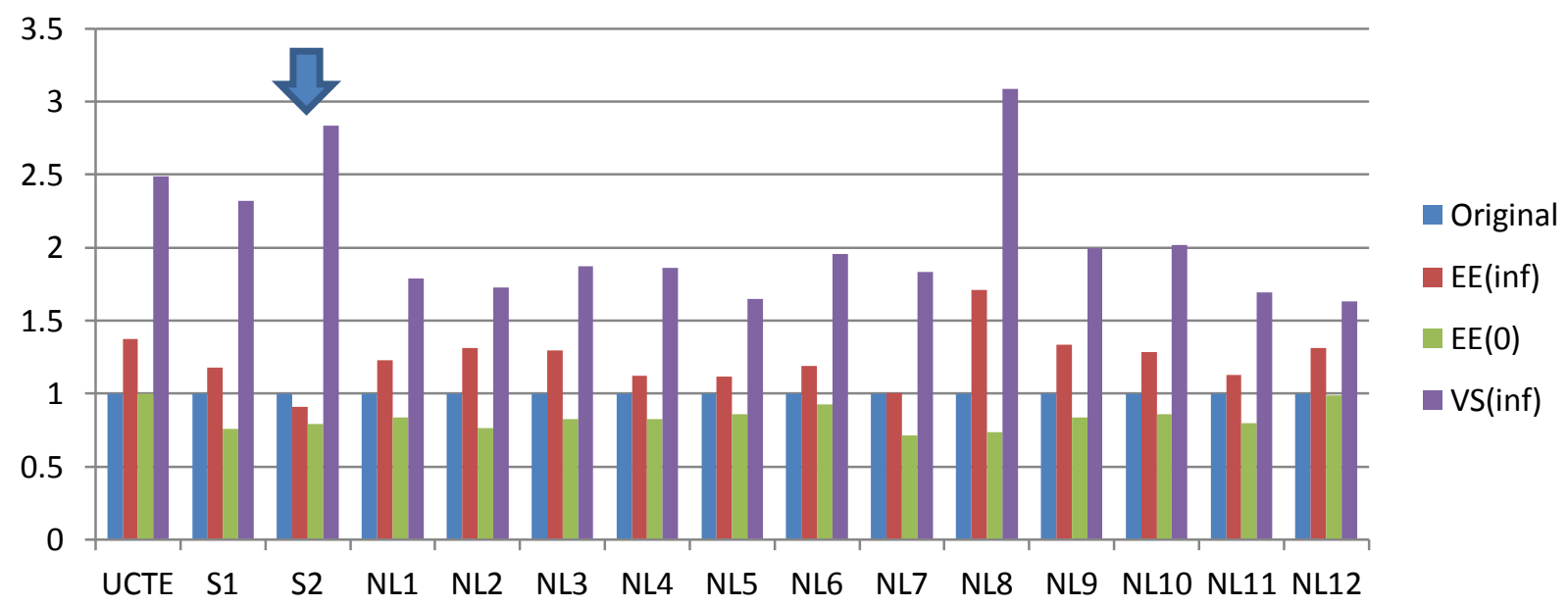

Fig. 1. Wiring costs (normalized) of networks obtained by fully random vertex and edge shuffling. All networks present optimal or almost optimal configurations for $\mathrm{EE}(0)$ except $\mathrm{S} 2$, which is not optimal under EE(inf) as well. UCTE transmission network has been used for comparison purposes. 


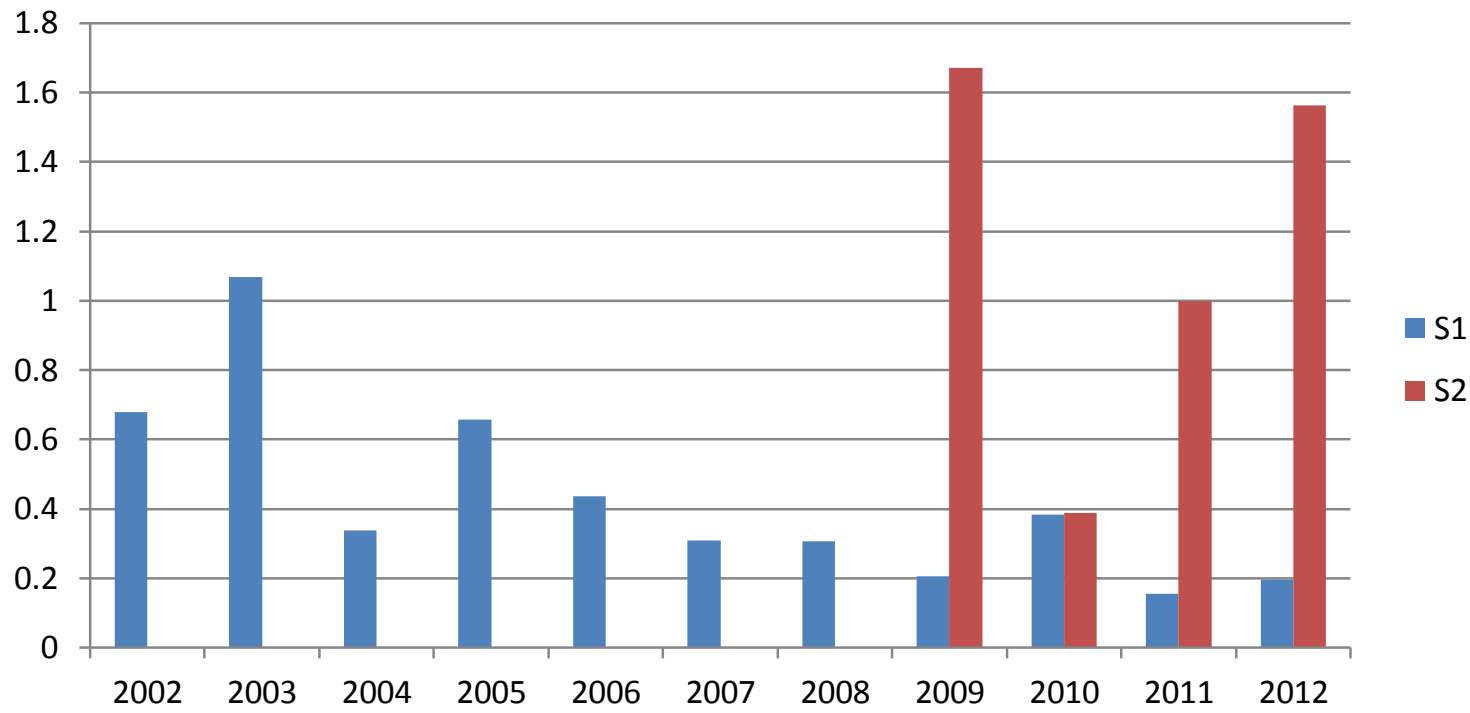

Fig. 2. Evolution of TIEPI values for S1 and S2 distribution networks.

For this study, we have had access to electric distribution quality data for only two distribution networks: S1 and S2. Here we use TIEPI values to measure the quality of electricity supply. TIEPI index, Spanish acronym for equivalent time of interruption of the installed capacity in medium voltage (i.e., $1 \mathrm{kV}<\mathrm{V}<36 \mathrm{kV}$ ) and similar to the English SAIDI, known as System Average Interruption Duration Index, is a numerical index that measures the effect of number and/or duration of interruptions affecting customers longer than three minutes. It is defined as:

$$
\operatorname{TIEPI}(S A I D I)=\frac{\sum U_{i} N_{i}}{N_{T}}
$$

where $N_{i}$ is the number of customers and $U_{i}$ is the annual outage time for location $i$, and $N_{T}$ is the total number of customers served. Figure 2 shows TIEPI values for distribution networks S1 and S2 and for several years. As we can observe, distribution network $\mathrm{S} 2$, which was previously noted as non-optimal from two of the three shuffling methods used, accumulates higher values of TIEPI for all years of data available, suggesting a lower performance for this distribution system.

\section{DisCuSSION AND CONCLUSIONS}

In this paper we investigate the effect of the wiring cost in the spatial organization of a sample of power distribution networks by means of shuffling the networks in systematic ways. We show that although they share similar topologies, suboptimal networks (i.e., those with topologies not organized to reduce the wiring cost) seem to accumulate more failures. This result is far from conclusive due to the lack of statistical significance of the sample. It is remarkable though that with these two complete datasets (i.e., S1 and S2) in terms of spatially defined topology and electric quality indexes, two distinguishable behaviors appear, with the one more prone to failures linked to a non-optimal topology.
It is an obvious conclusion that to finally predict how these systems respond to failures or dynamical variations, and how performance is linked with topology and optimal design of networks, more and better data processing is needed. Although topologies are statically similar, distribution networks have different objectives from transmission ones. The distribution grid has to deal with the last miles of the connectivity of the users and efficiency and costs are the first imperatives. The transmission network has to have more focus on the reliability of the network since it has to serve big regions and millions of consumption endpoints. It is also more difficult to trace failure data for specific distribution networks compared to transmission grids. In fact, the operations tend to aggregate failure data at larger regional or national level thus posing even more difficulties for data-driven optimization. Our research goal in the near term is (a) to have access to more data sources and distribution networks to provide a more sound statistical analysis to the promising results of this work, and (b) developing more specific topological (i.e. spatial) and extended metrics, involving electrical engineering characteristics of the network, to be able to characterize the kind of trade-off presented in this communication.

\section{ACKNOWLEDGMENT}

This research has been supported by the Next Generation Infrastructures (NGI) Foundation, research project grant number 05.24. G.A.P. is supported by the IBM $\mathrm{PhD}$ Fellowship 2013-2014.

\section{REFERENCES}

[1] M. E. J. Newman, Networks. An introduction. Oxford; New York: Oxford University Press, 2010, p. 720.

[2] G. A. Pagani and M. Aiello, "The Power Grid as a Complex Network: a Survey,” Physica A, May 2013.

[3] R. Albert, I. Albert, and G. L. Nakarado, "Structural Vulnerability of the North American Power Grid,” Phys. Rev. E, vol. 69, no. 2, p. 25103, 2004 
[4] P. Crucitti, V. Latora, and M. Marchiori, "Locating critical lines in high - voltage electrical power grids," Fluct. Noise Lett., vol. 5, no. 2, pp. L201-L208, Jun. 2005.

[5] R. Solé, M. Rosas-Casals, B. Corominas-Murtra, and S. Valverde, "Robustness of the European power grids under intentional attacks," Phys. Rev. E, vol. 77, no. 2, p. 26102, 2008.

[6] E. Bompard, E. Pons, and D. Wu, "Analysis of the structural vulnerability of the interconnected power grid of continental Europe with Integrated Power System and Unified Power System based on extended topological approach," Euro. Trans. Electr. Power, vol. 23, no. 5, pp. 620-637, 2012

[7] G. A. Pagani and M. Aiello, "Towards Decentralization: A Topological Investigation of the Medium and Low Voltage Grids," Smart Grid, IEEE Trans., vol. 2, no. 3, pp. 538-547, 2011.

[8] M. Barthelemy, "Spatial networks," Phys. Rep., vol. 499, no. 1-3, pp. 1-101, 2011.

[9] D. Jungnickel, "Graphs, networks and algorithms," Algorithm Comput. Math., vol. 5, 1999.
[10] R. K. Ahuja, T. L. Magnanti, and J. B. Orlin, Network Flows. Prentice Hall, New Jersey, 1993.

[11] Huneault M. and G. F.D., "A survey of the optimal power flow literature,” IEEE Trans. Power Syst., vol. 6, no. 2, pp. 762-770, 1991

[12] B. M.E. and W. F.F., "Network reconfiguration in distribution systems for loss reduction and load balancing," IEEE Trans. Power Deliv., vol. 4, no. 2, pp. 1401-1407, 1989.

[13] M. Rosas-Casals and R. Solé, "Analysis of major failures in Europe's power grid,” Int. J. Electr. Power Energy Syst., 2011.

[14] Y.-Y. Ahn, H. Jeong, and B. J. Kim, "Wiring cost in the organization of a biological neuronal network," Physica A, vol. 367, pp. 531-537, 2006

[15] D. Watts, Small Worlds: The Dynamics of Networks Between Order and Randomness, Princeton University Press, 1999.

[16] S. Maslov, K. Sneppen, Science 296 (2002) 910.

[17] C. Cherniak, J. Neurosci. 14 (1994) 2408. 